\title{
Substructure of Human von Willebrand Factor
}

Walter E. Fowler, Larry J. Fretto, Karen K. Hamilton, Harold P. Erickson, and Patrick A. McKee

The Howard Hughes Medical Institute Laboratories and Departments of Anatomy, Medicine, and Biochemistry, Duke University Medical Center, Durham, North Carolina 27710

\begin{abstract}
Using electron microscopy, we have visualized the substructure of human von Willebrand factor (vWf) purified by two different approaches. vWf multimers, which appear as flexible strands varying in length up to $2 \mu \mathrm{m}$, consist of dimeric units (protomers) polymerized linearly in an end-to-end fashion through disulfide bonds. Examination of small multimers (e.g., one-mers, two-mers, and three-mers) suggests that each protomer consists of two large globular end domains $(22 \times 6.5 \mathrm{~nm})$ connected to a small central node $(6.4 \times 3.4 \mathrm{~nm})$ by two flexible rod domains each $\sim 34 \mathrm{~nm}$ long and $\sim 2 \mathrm{~nm}$ in diameter. The protomer is $120 \mathrm{~nm}$ in length when fully extended. These same structural features are seen both in $\mathrm{vWf}$ molecules that were rapidly purified from fresh plasma by a new two-step procedure and in those purified from lyophilized intermediate-purity Factor VIII/vWf concentrates. The $240,000-\mathrm{mol}$ wt subunit observed by gel electrophoresis upon complete reduction of vWf apparently contains both a rod domain and a globular domain and corresponds to one half of the protomer. Two subunits are disulfide-linked, probably near their carboxyl termini, to form the protomer; disulfide bonds in the amino-terminal globular ends link protomers to form vWf multimers. The vWf multimer ștrands have at least two morphologically distinct types of ends, which may result from proteolytic cleavage in the globular domains after formation of large linear polymers. In addition to releasing fragments that were similar in size and shape to the repeating protomeric unit, plasmic degradation of either preparation of $\mathrm{vWf}$ reduced the size of multimers, but had no detectable effect on the substructure of internal protomers.
\end{abstract}

\section{Introduction}

Human Factor VIII/von Willebrand factor (FVIII/vWf) ${ }^{1}$ is a glycoprotein complex that corrects the plasma defects in both classic hemophilia A and von Willebrand's disease (1-3). The FVIII and vWf activities of the purified complex can be separated under a variety of nonphysiologic conditions; vWf activity is associated with $99 \%$ of the protein, while FVIII procoagulant activity derives from the other $1 \%(1,3)$. The vWf glycoprotein is synthesized by vascular endothelial cells and megakaryocytes

Dr. McKee is an Investigator of the Howard Hughes Medical Institute. Address correspondence to Dr. McKee, Howard Hughes Medical Institute/Duke University Medical Center, Box 3287, Durham, NC 27710. Received for publication 30 October 1984.

1. Abbreviations used in this paper: CTA, Committee on Thrombolytic Agents; DFP, diisopropylfluorophosphate; FVIII, Factor VIII; $\mathbf{K}_{\mathbf{A V}},\left(\mathrm{V}_{\mathbf{e}}\right.$ $\left.-V_{0}\right) /\left(V_{t}-V_{0}\right)$, where $V_{e}$ is elution volume, $V_{0}$ is void volume, and $V_{t}$ is total volume of the column; KIU, kallikrein inhibitor units; PAGE, polyacrylamide gel electrophoresis; vWf, von Willebrand factor.

J. Clin. Invest.

(c) The American Society for Clinical Investigation, Inc.

$0021-9738 / 85 / 10 / 1491 / 10 \$ 1.00$

Volume 76, October 1985, 1491-1500
$(4,5)$ and circulates in plasma as a series of multimers with molecular weights ranging from 0.5 million to 12 million (6-13).

The nonreduced structure seen on sodium dodecyl sulfate (SDS)-agarose gel electrophoresis is a series of logarithmicallyspaced bands, suggesting that each multimer differs from adjacent ones by a constant molecular weight of $\sim 500,000$ (14). SDSgel electrophoresis of reduced samples of purified $\mathrm{vWf}$ yields a single subunit of $\sim 240,000 \mathrm{~mol}$ wt $(8,15,16)$. Recent characterization of the cyanogen bromide fragments of carboxymethylated vWf (17) provides additional strong evidence that the circulating forms of $\mathrm{vWf}$ are polymers composed of a single type of subunit. Thus the 500,000 -mol wt protomer that corresponds to the main increment in multimer size is probably a dimer of the 240,000 -mol wt subunit.

Some investigators have shown on SDS-agarose gel electrophoresis that under certain conditions, each multimer of nonreduced $\mathrm{vWf}$ appears as a triplet composed of faint "side bands" above and below the most intense central band $(13,18,19)$. Ruggeri and Zimmerman (13) suggested that simple linear polymerization of a single protomer cannot explain these results. More recently these same investigators have reported resolution of the triplet into two major bands and four minor bands (20).

Ohmori et al. (21) have shown that vWf purified from FVIII/ $v W f$ concentrates appears in the electron microscope as strands of various lengths, consistent with the size heterogeneity shown by SDS-agarose gel electrophoresis. Although the presence of multiple globular domains along the length of each vWf strand was noted, no regularly repeating subunit structure was reported. In the present study we have examined $v W f$ multimers purified from fresh human plasma and from FVIII/vWf concentrates and have found a consistent repeating substructure, which we identify as the $500,000-\mathrm{mol}$ wt protomer.

\section{Methods}

Materials. Intermediate-purity human FVIII/vWf concentrates and platelet-rich plasma were obtained from the American Red Cross. Ristocetin was purchased from $\mathrm{H}$. Lundbeck and $\mathrm{Co}$. (Copenhagen, Denmark) and SeaKem agarose was obtained from MCI Biomedical (Rockland, MD). Carrier-free ${ }^{125} \mathrm{I}-\mathrm{Na}$ was from Amersham Corp. (Arlington Heights, IL). Nitrocellulose paper $(0.45 \mu \mathrm{m})$, Biogel A-15m, and Biogel A-150m were purchased from Bio-Rad Laboratories (Richmond, CA). Sephacryl S-1000 superfine was from Pharmacia Fine Chemicals (Piscataway, NJ) and heparin-agarose was from P-L Biochemicals Inc. (Milwaukee, WI). Sepharose-bound lactoperoxidase was obtained from Millipore Corp. (Bedford, MA). Diisopropylfluorophosphate (DFP) was from Sigma Chemical Co. (St. Louis, MO) and aprotonin (Trasylol) was obtained at 10,000 kallikrein inhibitor units (KIU) per ml from FBA Pharmaceuticals (New York, NY). All other chemicals were reagent grade or better and were used without further purification.

Purification of $v W f$. After receiving informed consent from apparently normal healthy volunteers, blood was drawn by the two-syringe technique into $1 / 10$ volume $3.8 \%$ sodium citrate and quickly mixed with aprotinin to give a final concentration of $100 \mathrm{KIU} / \mathrm{ml}$. The plasma was prepared by centrifugation at $2,510 \mathrm{~g}$ for $30 \mathrm{~min}$ at $15^{\circ} \mathrm{C}$ and then made $5 \mathrm{mM}$ with DFP (prediluted in Tris-saline buffer). Some plasma samples were 
then treated with additional aprotinin and with EDTA to give final concentrations of $200 \mathrm{KIU} / \mathrm{ml}$ and $5 \mathrm{mM}$, respectively. All plasma samples were then immediately applied to Sephacryl S-1000 $(5.0 \times 83 \mathrm{~cm})$ equilibrated in $0.15 \mathrm{M} \mathrm{NaCl}-0.05 \mathrm{M}$ Tris- $0.02 \%$ sodium azide, $\mathrm{pH} 7.35$, and eluted at $150 \mathrm{ml} / \mathrm{h}$ into $12.3-\mathrm{ml}$ fractions. Fractions were analyzed by SDS-polyacrylamide gel electrophoresis (PAGE) (silver-stained) and assayed for ristocetin cofactor activity as described previously (21). Appropriate pools were applied to heparin-agarose $(1.6 \times 2.6 \mathrm{~cm})$ equilibrated in $0.2 \mathrm{M}$ ammonium formate, $\mathrm{pH}$ 7.3. After sample application the column was washed with $10 \mathrm{vol}$ of $0.2 \mathrm{M}$ ammonium formate, $\mathrm{pH}$ 7.3. $\mathrm{vWf}$ was then eluted from the column in the reverse direction using a linear gradient ( $50 \mathrm{ml}$ total) from 0 to $0.6 \mathrm{M} \mathrm{NaCl}$ in $0.2 \mathrm{M}$ ammonium formate. Fractions were analyzed by SDS-PAGE, ristocetin-induced platelet agglutination, electron microscopy, and SDS-agarose gel electrophoresis followed by radioimmunoblotting (described below).

FVIII/vWf was also isolated from Red Cross antihemophilic factor concentrates by selective precipitation using polyethylene glycol 4000 followed by gel filtration on $4 \%$ agarose (Biogel A-15m) as previously described (22). Protein concentrations were determined using a commercial protein assay (Bio-Rad Laboratories). Rechromatography in $\mathbf{0 . 2 5}$ $\mathrm{M} \mathrm{CaCl}_{2}-0.10 \mathrm{M} \mathrm{NaCl}-0.01 \mathrm{M}$ L-histidine- $0.001 \mathrm{M}$ benzamidine- $\mathrm{HCl}$, pH 6.0 , on Biogel A-15m columns $(2.6 \times 90 \mathrm{~cm})$ yielded $v W f$ that was free of FVIII (23). Additional fractionation of $\mathrm{vWf}$ multimer populations was achieved on $1 \%$ agarose (Biogel A-150m; $1.6 \times 52 \mathrm{~cm}$ ) in $0.2 \mathrm{M}$ ammonium formate. vWf pools were usually concentrated between gel filtration steps by precipitation with polyethylene glycol 4000 (12\% final concentration) at $4^{\circ} \mathrm{C}$; after centrifugation at $3,800 \mathrm{~g}$ and $4^{\circ} \mathrm{C}$ the pellet was redissolved $(3-5 \mathrm{mg} / \mathrm{ml})$ and dialyzed in $0.15 \mathrm{M} \mathrm{NaCl}-0.05 \mathrm{M}$ Tris,

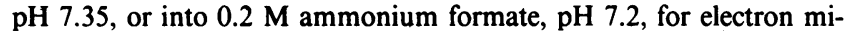
croscopy.

Plasmin digestion of $v W f$. Fractions of $\mathrm{vWf}$ eluted from Biogel A$150 \mathrm{~m}$ at $\sim 0.2 \mathrm{mg} / \mathrm{ml}$ were incubated in $0.2 \mathrm{M}$ ammonium formate$0.02 \%$ sodium azide at $37^{\circ} \mathrm{C}$ with freshly prepared human plasmin (24) added in increments of 0.02 CTA units $(0.8 \mu \mathrm{g})$ per $\mathrm{ml}$ of incubation mixture at $0,3,48$, and $60 \mathrm{~h}$ to give a final plasmin-to-vWf subunit mole ratio of 1 to 23 . Aliquots were removed at $0,1,6,18,26$, and $70 \mathrm{~h}$, treated with aprotonin, and prepared for electron microscopy.

A native preparation of $\mathrm{vWf}(0.2 \mathrm{mg} / \mathrm{ml})$ eluted from heparin-agarose was also digested with freshly-activated plasmin (1.5 CTA U/ml final concentration) at $37^{\circ} \mathrm{C}$ in $0.2 \mathrm{M}$ ammonium formate- $0.25 \mathrm{M} \mathrm{NaCl}, \mathrm{pH}$ 7.3. Aliquots of the reaction mixture were removed at various times between $3 \mathrm{~min}$ and $8 \mathrm{~h}$, were treated with aprotinin $(670 \mathrm{KIU} / \mathrm{CTA}$ unit) to terminate the digestion, and were analyzed by electron microscopy and SDS-agarose gel electrophoresis followed by radioimmunoblotting.

Electrophoretic analysis. SDS-5\% PAGE was performed by the method of Laemmli (25). Analysis of nonreduced samples was performed by SDS electrophoresis on $1.25 \%$ and $0.8 \%$ agarose gels in a Tris-borate buffer system at pH $8.6(25)$. A constant current of $5 \mathrm{~mA}$ per slab $(0.15$ $\times 14 \times 14 \mathrm{~cm}$ ) was used for the first $40 \mathrm{~min}$ and then $14 \mathrm{~mA}$ per slab for $3 \mathrm{~h}$. The gels were stained with Coomassie Blue as described previously (21) or were electrophoretically transferred to nitrocellulose paper and incubated with ${ }^{125}$ I-labeled rabbit antihuman vWf IgG, followed by radioautography (23). Some polyacrylamide gels were silver-stained by the procedure of Merril et al. (26).

Electron microscopy. Samples for electron microscopy were prepared using the glycerol spray/vacuum dry method described by Fowler and Erickson (27), which was also used by Ohmori et al. (21) to visualize vWf. Specimens were examined in an electron microscope (model 300; Philips Electronic Instruments, Inc., Mahwah, NJ) at $80 \mathrm{kV}$ accelerating voltage. All micrographs were recorded at a magnification of 23,000 or 49,500 . The magnification of the electron microscope was calibrated using negatively stained crystals of catalase. The micrographs are printed such that the platinum grains appear dark; final magnification is indicated in the figure legends.

\section{Results}

To avoid the loss of large multimers that can occur during purification of $\mathrm{vWf}$ from plasma and to minimize chances of de- naturation, we developed a new purification protocol involving only two steps. First, gel filtration on Sephacryl S-1000 of freshly drawn citrated plasma, in the absence of EDTA or heparin, clearly separated the vWf ristocetin cofactor activity from virtually all of the plasma protein (Fig. $1 A$ ). This peak contained $>75 \%$ of the activity applied to the column. The vWf multimers were detected by radioimmunoblots and fractionated by size in this step (Fig. $1 A$, inset): the largest eluted just after the void volume $\left(\mathrm{K}_{\mathrm{Av}}=0.13\right)$ and progressively smaller multimers eluted in successive fractions as far as $2 \mathrm{~V}_{0}$. Peak vWf activity and concentration were at $\mathrm{K}_{\mathrm{Av}}=0.31$. In the second step, the large $\mathrm{vWf}$ multimers in Pool I (Fig. $1 A$ ) were concentrated and further purified by adsorption on heparin-agarose and then elution with a salt gradient; as shown in Fig. $1 B$ the vWf eluted between 0.2 and $0.3 \mathrm{M} \mathrm{NaCl}$. The yield of $\mathrm{vWf}$ ristocetin cofactor activity in this step was $\sim 70 \%$ and none was detected in the breakthrough peak or in the wash before the $\mathrm{NaCl}$ gradient. The radioim-

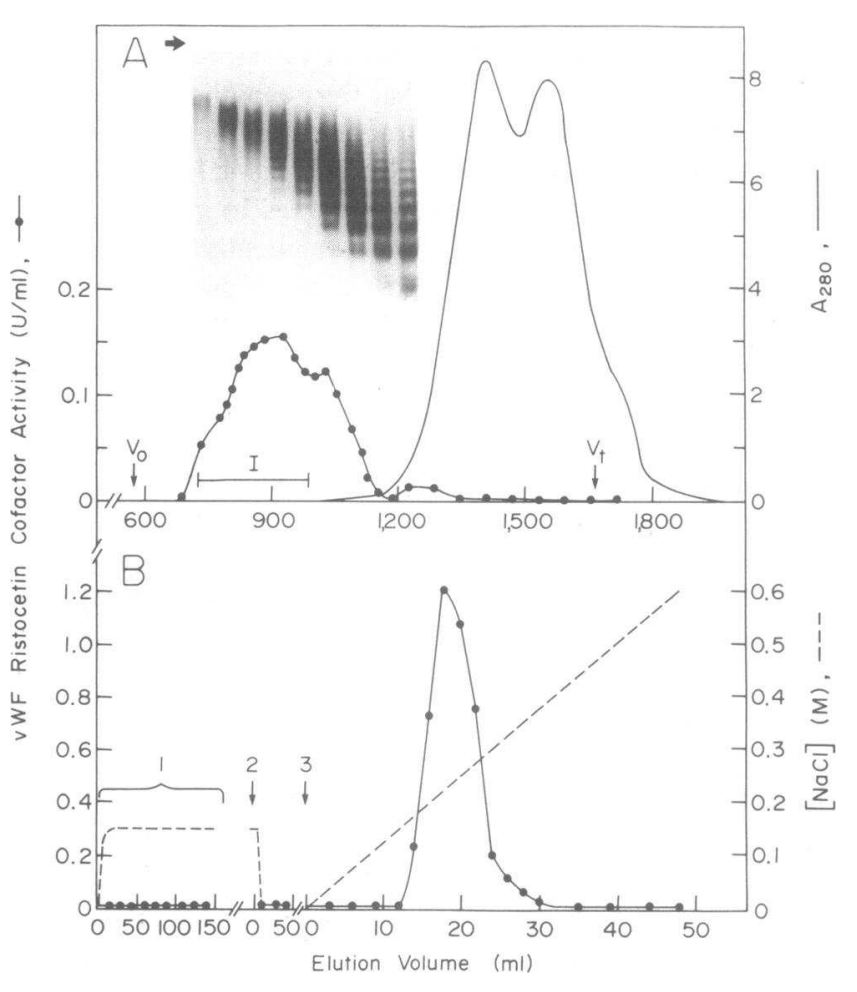

Figure 1. Purification of large vWf multimers from human plasma. (A) Elution profiles from Sephacryl S-1000 $(5.0 \times 83 \mathrm{~cm})$ of absorbance at $280 \mathrm{~nm}\left(\mathrm{~A}_{280}\right)$ and of vWf ristocetin-induced platelet-agglutinating activity (ristocetin cofactor activity; relative to $1 \mathrm{U} / \mathrm{ml}$ for whole normal plasma) after applying $60 \mathrm{ml}$ of plasma (containing 5 $\mathrm{mM} \mathrm{DFP}$ and $100 \mathrm{KIU} / \mathrm{ml}$ aprotinin) that was prepared from freshly drawn citrated blood. The column was equilibrated in $0.05 \mathrm{M}$ Tris$0.15 \mathrm{M} \mathrm{NaCl}-0.02 \%$ sodium azide, $\mathrm{pH} 7.35$, and eluted at $150 \mathrm{ml} / \mathrm{h}$ into 12.3-ml fractions; the void volume $\left(\mathrm{V}_{0}\right)$ and total volume $\left(\mathrm{V}_{\mathrm{t}}\right)$ of the column are indicated. Inset, Radioimmunoblot of SDS- $0.8 \%$ agarose gel electrophoresis (arrow indicates top of lanes) of samples from every fifth fraction between fractions 60 and 100, inclusive. The center of each gel lane is aligned with the elution volume of the corresponding fraction. $(B)$ Elution profile from heparin-agarose $(1.6 \times 2.6 \mathrm{~cm})$ of $\mathrm{vWf}$ ristocetin cofactor activity during: (1) application of half the volume of each fraction in Pool I (horizontal bar in $A$ ) from the S1000 column, (2) wash with $0.2 \mathrm{M}$ ammonium formate, $\mathrm{pH} 7.3$, and (3) elution with a linear gradient of $0-0.6 \mathrm{M} \mathrm{NaCl}$ in $0.2 \mathrm{M}$ ammonium formate. The column was eluted at $50 \mathrm{ml} / \mathrm{h}$ and $1-\mathrm{ml}$ fractions were collected during the $\mathrm{NaCl}$ gradient. $\mathrm{A}_{280}$ was less than 0.02 . 
munoblots in Fig. $2 \mathrm{~A}$ show that the largest vWf multimers (14mers to 20-mers) found in plasma (lane $P$ ) were preserved in the vWf eluted from heparin-agarose (lane 1); we term this the "native preparation" of vWf. We also purified vWf from FVIII/ $v W f$ concentrates by published procedures $(22,23)$; the fraction used lacked the largest vWf multimers and contained predominantly three-mers, four-mers, and five-mers (Fig. $2 A$, lane 2). As seen by SDS-PAGE of reduced vWf samples (Fig. $2 B$ ), the native preparation (lane 1) contained a single $240,000 \mathrm{~mol} \mathrm{wt}$ polypeptide with $<2 \%$ proteolytically cleaved subunit, while the material prepared from lyophilized concentrates (lane 2 ) contained a small amount $(<13 \%)$ of degraded subunit at 160,000 mol wt in addition to the major band at $240,000 \mathrm{~mol} w t$.

In the electron microscope, both preparations were seen to contain the same type of coiled filamentous structures, also seen by Ohmori et al. (21). As in that study, no more than two definitive ends were seen for a given strand, which means that the strands are linear and not branched. Fig. $3 A$ shows examples of long, straight vWf molecules from the native preparation, in which the presence of a repeating substructure could be appreciated. The most obvious repeating features are large and small nodular domains, which alternate along the length of the vWf multimer and are separated by thin, flexible rod segments of relatively constant length. The sections of the three molecules shown in Fig. $3 \mathrm{~A}$ are approximately in register with respect to this period. One of these vWf molecules was the longest we have observed by electron microscopy: it extended over $1,800 \mathrm{~nm}$

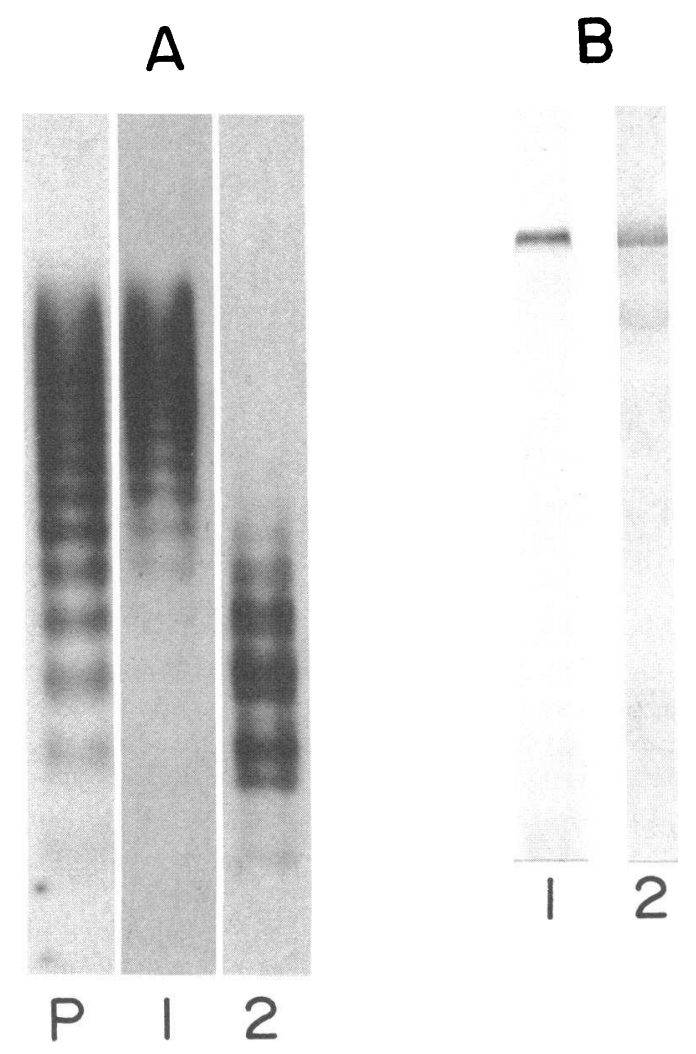

Figure 2. SDS-gel electrophoretic comparison of vWf preparations. $(A)$ Radioimmunoblots of SDS- $0.8 \%$ agarose gel of: $P$, whole plasma; 1 , $\mathrm{vWf}$ purified from fresh plasma as in Fig. 1; and 2, vWf purified from lyophilized FVIII/vWf concentrates (23). (B) SDS-5\% PAGE of vWf preparations $l$ and 2 described above, stained with Coomassie Blue. and comprised 17 repeating units. As indicated in Fig. $2 \mathrm{~A}$, the average length of vWf multimers purified from FVIII/vWf concentrates was much shorter than that of molecules from the native preparation. However, as shown in Fig. $3 B$, straight stretches of these smaller vWf molecules exhibit the very same repeating features as those of the "native" molecules seen in Fig. $3 \mathrm{~A}$. Also shown in Fig. $3 \mathrm{~B}$ is an example of a loosely coiled vWf molecule, which is representative of the most frequently observed structures in electron microscopic specimens from both types of $\mathrm{vWf}$ preparations. The coiled forms only occasionally showed a clear substructure, due to multiple overlaps. We therefore selected strands that were well extended for our structural analyses. Since it was noted that relatively short $\mathrm{vWf}$ multimers were more often found in an extended form in the electron microscopic specimens, we decided to use the original vWf preparation containing these smaller forms to isolate and visualize different multimer species (i.e., one-mers, two-mers, etc.).

Thus, the vWf preparation obtained from the FVIII/vWf concentrates was further fractionated by gel filtration on $1 \%$ agarose. Fig. 4 shows the resulting elution profile and the specific vWf ristocetin cofactor activity, which was fairly constant in the first half of the peak and decreased rapidly after fraction $e$, where the median multimer size dropped to four repeating units (see below). The multimer analysis of selected fractions by SDS-agarose gel electrophoresis and radioimmunoblotting is presented in Fig. 5. Although each fraction still contained a range of multimer sizes, the median molecular weight clearly decreased with increasing elution volume. Samples of fractions selected across the peak were prepared for electron microscopy and representative micrographs are shown in Fig. 6. In fields $A$ and $D$ the presence of rare contaminating fibrinogen molecules is noted for purposes of size and shape comparison. The individual nodules on the $\mathrm{vWf}$ filaments ranged in size from less than that of the fibrinogen $\mathrm{E}$ domain to larger than that of the fibrinogen $\mathrm{D}$ domains (see ref. 28). The trend of decreasing multimer size with elution volume (Fig. 5) is also clear in these micrographs. Comparison of different vWf molecules, particularly those with a minimum number of overlaps, revealed the repeating substructure along the entire length of the vWf multimers.

The structure of the repeating unit is clearly shown in the gallery of micrographs of small vWf multimers in Fig. 7. Molecules representing the smallest consistent structure observed are shown in the top row of Fig. $7 \mathrm{~A}$; examples of molecules two and three times this size are given in the middle and bottom rows of Fig. $7 A$, respectively. The repeating unit, which we call the $\mathrm{vWf}$ protomer, has a maximum length of $120 \mathrm{~nm}$ and appears to be a dimer with twofold symmetry about a central axis. Each of the two identical subunits consists of a long, thin flexible rod with a large globular domain at one end. A smaller globular domain is usually seen in the center of the protomer where the rod domains of the two subunits are joined. Of note, we never observed multimers terminating with this small nodular domain, which makes it highly unlikely that the large globular regions constitute the center of the protomer. In many instances both rod domains were fairly linear near the central node of the protomer, forming an angle that ranged from $20^{\circ}$ to $180^{\circ}$ and averaged $77^{\circ}$. The best examples of vWf multimers in which the protomers were all nearly maximally extended (i.e., having a central angle of $180^{\circ}$ ) are shown in Fig. $7 \mathrm{~B}$ and $C$. The multimers in Fig. $7 B$ were purified from FVIII/vWf concentrates, while that in Fig. $7 C$ was from our "native preparation." The structure of the protomers within these multimers is essentially identical 

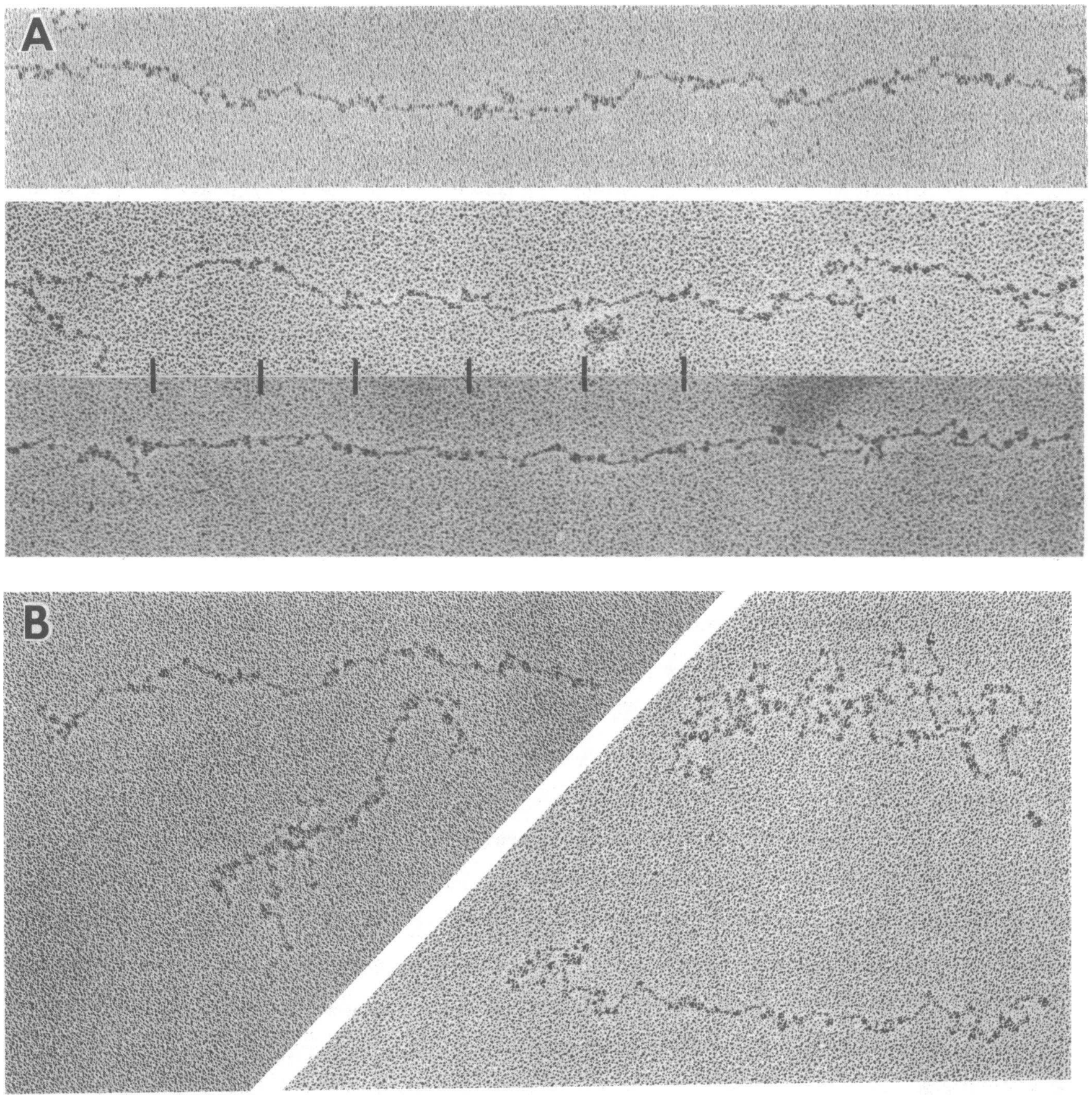

Figure 3. Electron micrographs of vWf molecules from: $(A)$ "native" preparations containing the largest multimers found in fresh plasma and $(B)$ conventional $\mathrm{vWf}$ preparations from lyophilized FVIII/vWf concentrates lacking very large multimers. The long, straight molecules in $A$ are shown approximately on register with respect to a $\sim 120$ $\mathrm{nm}$ repeat (see text); several repeats are marked by bars between the lower two molecules. The straight molecules shown in $B$ exhibit the

(apparent differences in contrast in the two images are normal variations in the electron microscope specimens).

A model for the consistent features of the protomer structure, as seen both in large and small multimers, is given in Fig. $7 \mathrm{D}$ together with the maximum measured dimensions for the various domains. The overall length of the fully extended protomer is $120 \mathrm{~nm}$, the rods being $34 \mathrm{~nm}$ and the globular domains $26 \mathrm{~nm}$ long for each subunit. The connection between the rod domains was usually discernible as a small central nodule, its average same $120 \mathrm{~nm}$ repeat seen in "native" $\mathrm{vWf}$ molecules. The coiled molecule (shown at the top right of $B$ ) that contains multiple crossovers, demonstrates the repeating substructure in places but no regular superstructure (i.e., packing). Note that such tangled molecules were the most frequently observed forms and probably represent the conformation in solution (See Discussion). Magnification $\times 150,000$.

dimensions being $6.4 \mathrm{~nm}$ by $3.4 \mathrm{~nm}(n=25) .^{2}$ As indicated in Fig. $7 D$, the large globular domains at either end of the protomer were often resolved into two or three nodular subdomains. The globular domains occurring at the free ends of multimers were

2. This and all subsequent values in the text have been corrected for the thickness of the platinum shell around the molecule by subtracting 4 $\mathrm{nm}$ from the actual measured values $(27,28)$. 


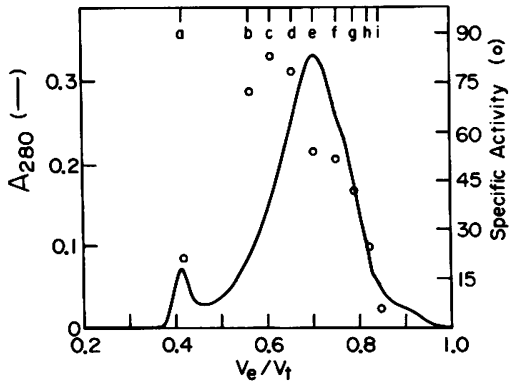

Figure 4. Absorbance elution profile of $\mathrm{vWf}$ rechromatographed on $1 \%$ agarose $(1.6 \times 52 \mathrm{~cm})$ in $0.2 \mathrm{M}$ ammonium formate, $\mathrm{pH}$ 7.0. Specific vWf activity (0) of fractions designated $a$ through $i$ by the ristocetin-induced platelet agglutination assay is given as $U / \mathrm{mg}$, where one unit is the activity contained in $1 \mathrm{ml}$ of pooled normal plasma.

heterogeneous in size and often did not show the typical protomer end structure (see below). Consequently, their dimensions were estimated by measuring the entire globular interprotomeric contact region within the multimers. Although this region was also variable in appearance, containing two, three, or four irregularly shaped nodules, it was fairly uniform in overall size. The area ( $\pm \mathrm{SD}, n=20$ ) of $t w o$ joined protomer ends was $255 \pm 23$ $\mathrm{nm}^{2}$ and the volume was $1,100 \pm 350 \mathrm{~nm}^{3}$, assuming an ellipsoidal shape. A structure of this size is equivalent to the combined volumes of three to four fibrinogen fragment D domains (28). The interprotomer junction within the multimer probably involves a small overlap of the protomeric globular end nodules.

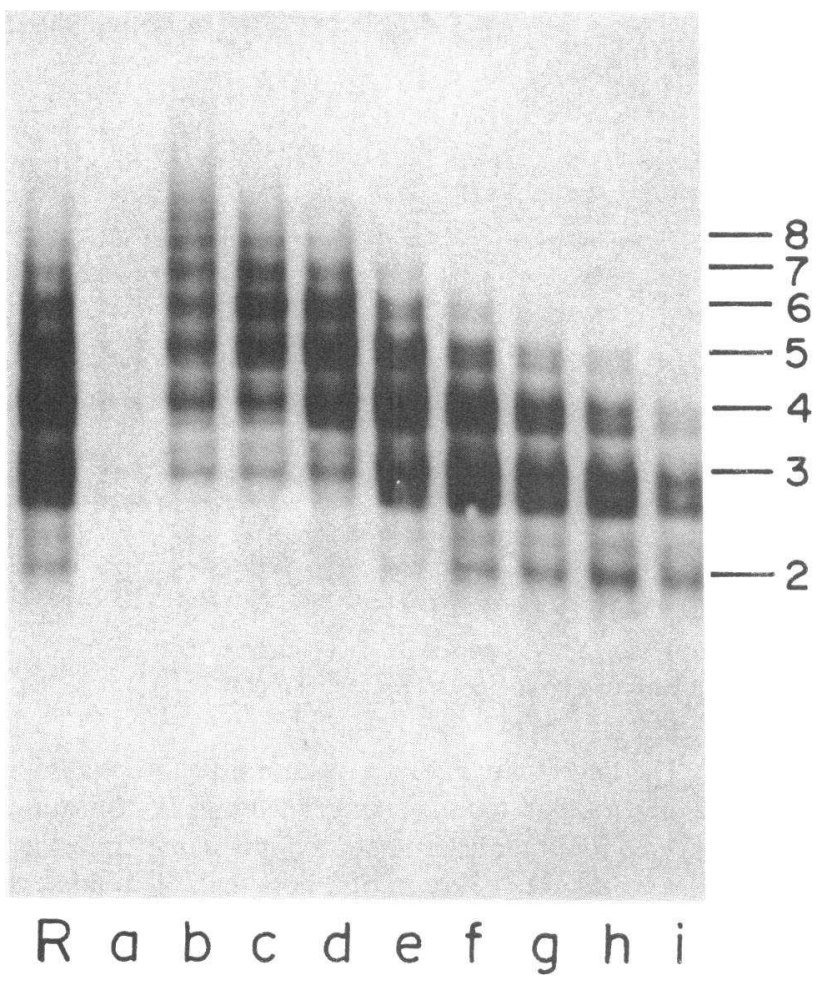

Figure 5. Autoradiograph of Western blot of SDS-1.25\% agarose gel of nonreduced $\mathrm{vWf}$ samples $(\sim 15 \mathrm{ng})$ from the fractions designated $a$ to $i$ in Fig. 4 after incubation with ${ }^{125}$ I-labeled rabbit anti-human vWf antibody. Precolumn sample is designated $R$. Multimer sizes for the major bands are identified by the number of repeating units (protomers) they contain, as indicated on the right.
To increase the percentage of smaller multimers in our preparations, in particular the number of individual protomers, we treated fraction $c$ from the $1 \%$ agarose column (Figs. 4 and 5) with the serine proteinase plasmin. Hamilton et al. (23) suggested that plasmin generates vWf fragments consisting of protomers or multiples of protomers that, before reduction, have lost only relatively small peptides. Not surprisingly, micrographs of specimens prepared from a 70-h digestion sample (Fig. 8 B) showed a striking reduction in the length of the $\mathrm{vWf}$ multimers; however, relative to control vWf (Fig. $8 \mathrm{~A}$ ), there was a remarkable preservation of the appearance of the vWf protomer, whether free or within a multimer. As shown in Fig. $8 \mathrm{~B}$, which contains mostly protomer-sized fragments, the central nodule and rod domains of the protomer structure appeared unaltered, whereas some of the globular end domains appeared slightly smaller.

In addition to the "normal" globular ends of the protomer structure depicted in Fig. $7 \mathrm{D}$, multimers from both types of our $\mathrm{vWf}$ preparations showed a range of end morphologies with two extreme variant forms: (a) virtual absence of a globular domain; and $(b)$ an elongated globular domain as large as the double domains formed by the fusion of internal protomers. Fig. 9 shows clear examples of these two variant end structures. Some multimers were found that had a different variant at each end, while in others both ends exhibited the same morphology. The end nodules could not always be delineated with precision so it was difficult to decide in many cases whether an end had a "normal" or a "variant" morphology. This is well illustrated by the multimer ends seen in Fig. 7.

The variant end morphologies could be explained if large multimers were occasionally fragmented at a point within a protomer near the junction of the rod and globular domains as shown by the thin arrows in Fig. 10. This type of model might also explain the triplet-band pattern of the multimers seen on SDS-agarose gel electrophoresis (13). Multimers composed of a given number of protomers, but having different combinations of end morphologies, would have slightly different molecular weights. As shown in Fig. 10, the simplest case of each "n-mer" having either a combination of variant ends or two normal ends would give rise to three bands separated by molecular weight intervals of $\sim 150,000$, with the central band being the most prominent. Bands with intermediate molecular weight spacing could be added to this triplet pattern if multimers having both one normal and one variant end are considered. Indeed, such additional bands were recently observed (20).

Since the variant end morphologies suggest a proteolytic origin, and since this model also suggests a relationship to the triplet-band pattern, we examined the effect of proteolysis on the electrophoretic pattern of vWf multimers. In our experience the triplet pattern was always pronounced for vWf in Red Cross FVIII/vWf concentrates, while our "native vWf preparation", like whole plasma, had only a very faint triplet pattern (Fig. 11, lanes $P$ and $a$ ). To determine if the serine proteinase plasmin could be responsible for the appearance of the triplet-band pattern we plasmin-digested a native $\mathrm{vWf}$ preparation. Although the multimer size was clearly decreased by plasmin (Fig. 11), the triplet pattern was not enhanced. As previous studies with multimers prepared from FVIII/vWf concentrates have suggested (23), the smaller multimers generated by plasmin have nearly the same spacing and mobilities as the corresponding undigested small vWf multimers of whole plasma. This indicates that the plasmin cleavage responsible for generating smaller multimers is near the interprotomer site (short broad arrows in Fig. 10) 

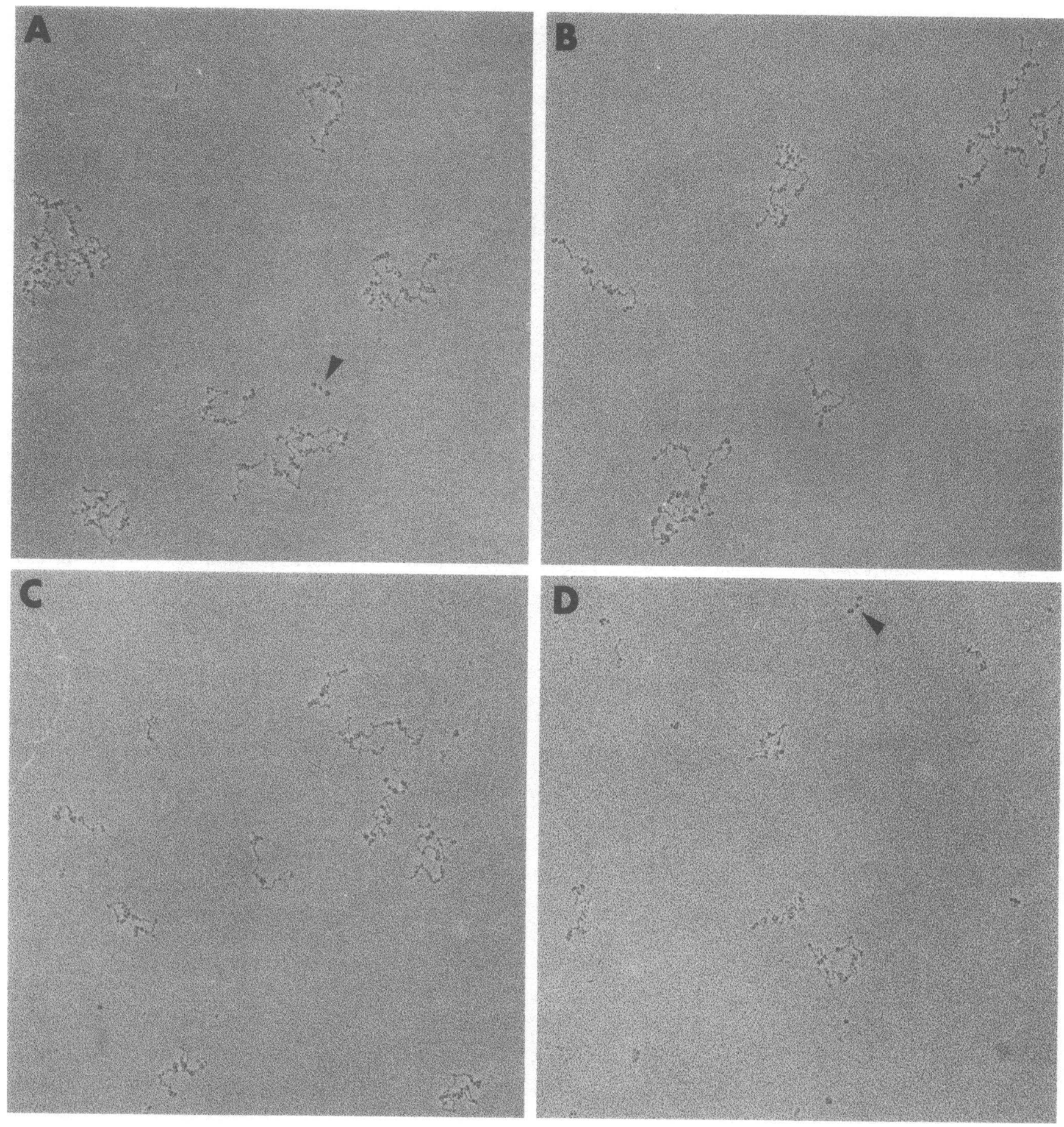

Figure 6. Electron micrographs $(\times 75,000)$ of rotary shadowed $\mathrm{vWf}$ from $1 \%$ agarose chromatography. Samples used in panels $A, B, C$, and $D$ were taken from fractions identified in Fig. 4 as $c, e, g$, and $i$, respectively. Arrows indicate fibrinogen molecules for size comparison.

and is different from that of the proteinase(s) acting in vivo and/ or in the preparation of FVIII/vWf concentrates.

\section{Discussion}

A previous electron microscope study from our laboratories showed that purified $\mathrm{vWf}$ has a flexible, filamentous structure with a length heterogeneity $(100-1,200 \mathrm{~nm})$ that reflected the molecular weight distribution of those preparations $\left(0.5-5 \times 10^{6}\right.$ mol wt) (21). Although the electron microscope preparation technique used in that study is ideal for visualizing large extended molecules (29), the substructure of the vWf multimers was not elucidated. The largest circulating vWf multimers were absent from those preparations and the relatively complex purification protocol allowed for potential alteration of the native multimer structure. Moreover, even before purification, the $\mathrm{vWf}$ multimers in the Red Cross FVIII/vWf concentrates showed a pronounced triplet pattern by radioimmunoblots.

We have now prepared from fresh plasma a representative fraction of large vWf multimers for comparison with the previous preparation. This was achieved using a purification scheme (Fig. 1) that was designed to minimize denaturation or proteolysis of the plasma vWf: no precipitation steps were used; the buffer $\mathrm{pH}$ and ionic strength were maintained near physiologic; proteinase 

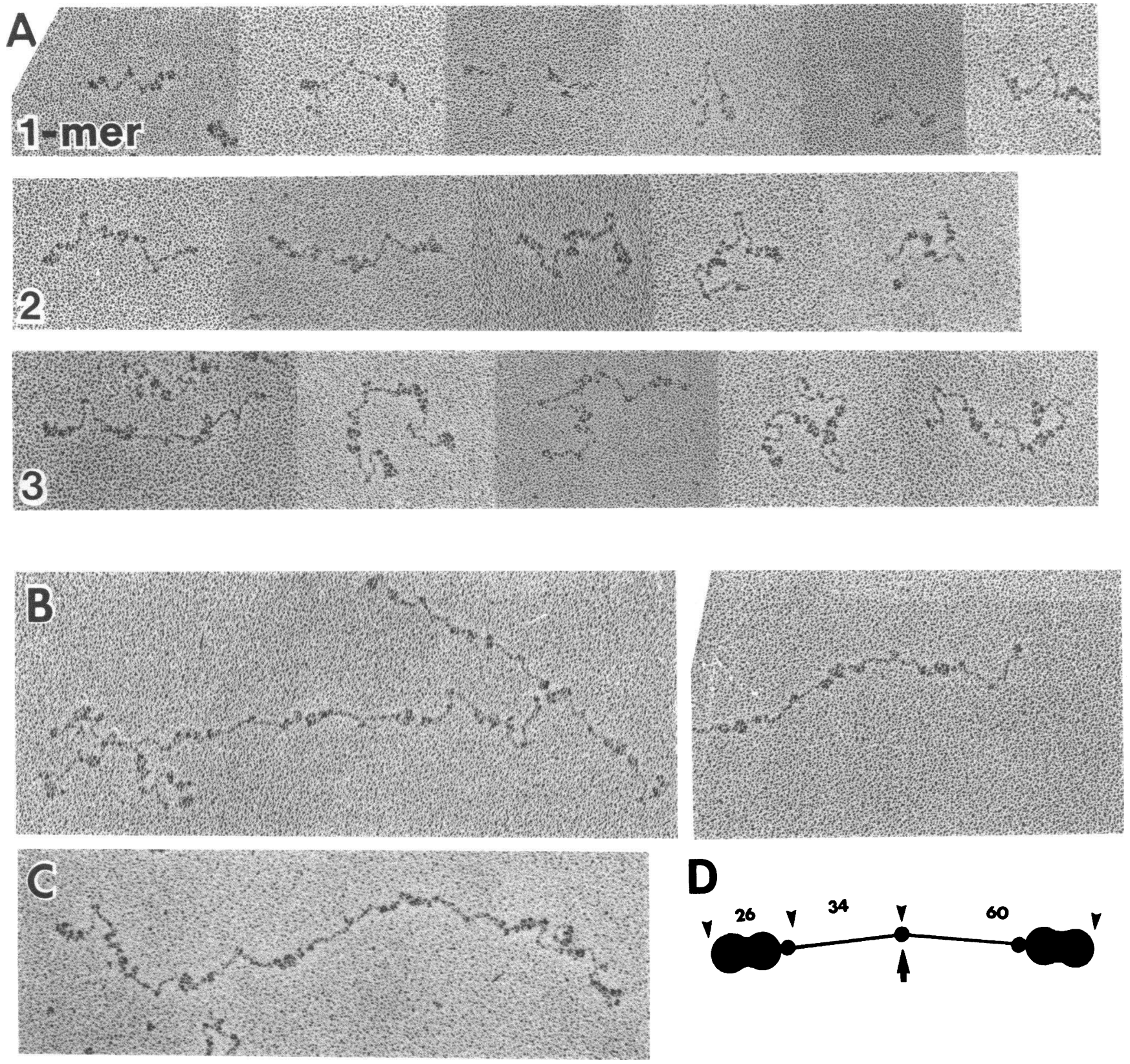

Figure 7. Electron micrographs $(\times 150,000)$ of selected $\mathrm{vWf}$ molecules demonstrate the repeating protomer structure. $(A)$ Top row shows examples of vWf molecules consisting of a single protomer ("1-mers"); middle row shows vWf molecules composed of two protomers ("2mers"); bottom row gives examples of $\mathrm{vWf}$ molecules three units in length ("3-mers"). (B) Examples of extended multimers from the conventional preparation showing many details of the protomer configu-

inhibitors were used; and final purification was achieved within $12 \mathrm{~h}$ of drawing the blood. The resulting large multimer preparation showed no evidence of degradation when analyzed by SDS-PAGE after reduction (Fig. $2 B$, lane 1 ) and by radioimmunoblots (Fig. $2 A$ ): the multimer positions were unaltered and the very faint sidebands of the triplet band pattern were not enhanced relative to that of plasma $\mathrm{vWf}$ (Fig. $2 \mathrm{~A}$, lane $\mathrm{P}$ ).

Electron microscope specimens of $\mathrm{vWf}$ samples from several preparations of both types (i.e., from fresh plasma and from FVIII/vWf concentrates) all contained examples of multimers in which the same repeating substructure was evident (Fig. 3). We selected the most extended molecules for our present analysis ration. $(C)$ Example of an extended multimer from the native preparation showing the same detailed protomer structure as seen in $B$. $(D)$ Schematic representation of vWf protomer showing the two subunits joined at central twofold symmetry axis (large arrow). Maximum dimensions in $\mathrm{nm}$ (uncorrected for replica thickness) of the rod and globular domains are indicated within the smaller arrows.

because they show the substructure most clearly. However, the most frequent and typical form of the vWf multimers in the electron microscope specimens was a loosely tangled coil, $\sim 100$ $300 \mathrm{~nm}$ in overall size. One example is shown in Fig. $3 B$ and another in Fig. $6 \mathrm{~A}$. Two observations suggest that these tangled forms are probably close to the conformation of the molecules in solution. The largest plasma vWf multimers (mol wt $>8 \times 10^{6}$ ) eluted from our Sephacryl S-1000 column slightly behind the void volume, at $\mathrm{K}_{\mathrm{Av}}=0.13$. The more average-sized multimers eluted later, the peak having $\mathrm{K}_{\mathrm{Av}}=0.31$. According to a recently published calibration of Sephacryl S-1000 (30) these elution positions correspond to those of spherical particles with 140 and 

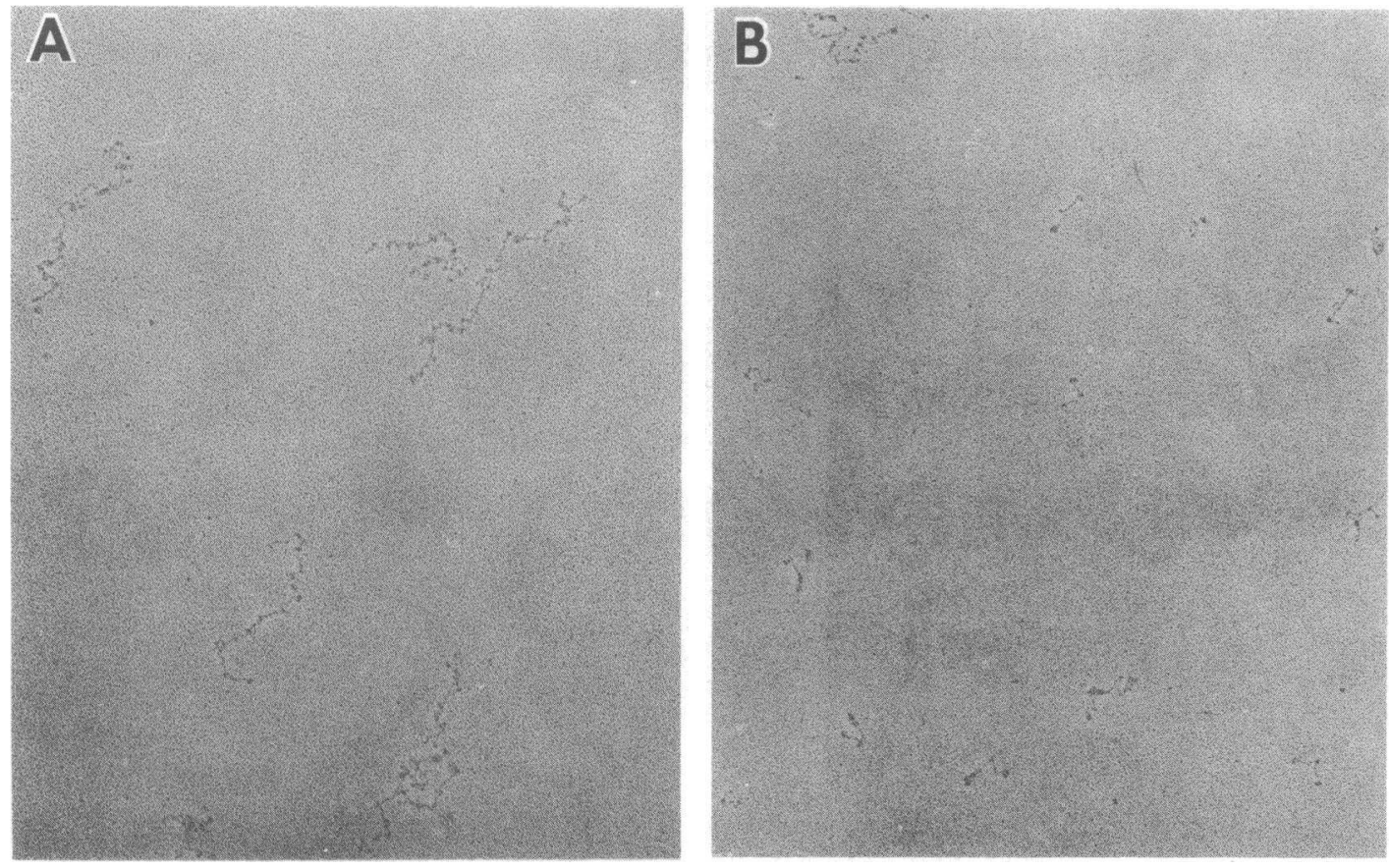

Figure 8. Electron micrographs $(\times 60,900)$ of rotary shadowed native and plasmin-digested vWf. $(A)$ Predigestion sample from fraction $c$ of Fig. 4. $(B) \mathrm{vWf}$ sample from 70 -h digestion, showing primarily protomer-sized fragments.

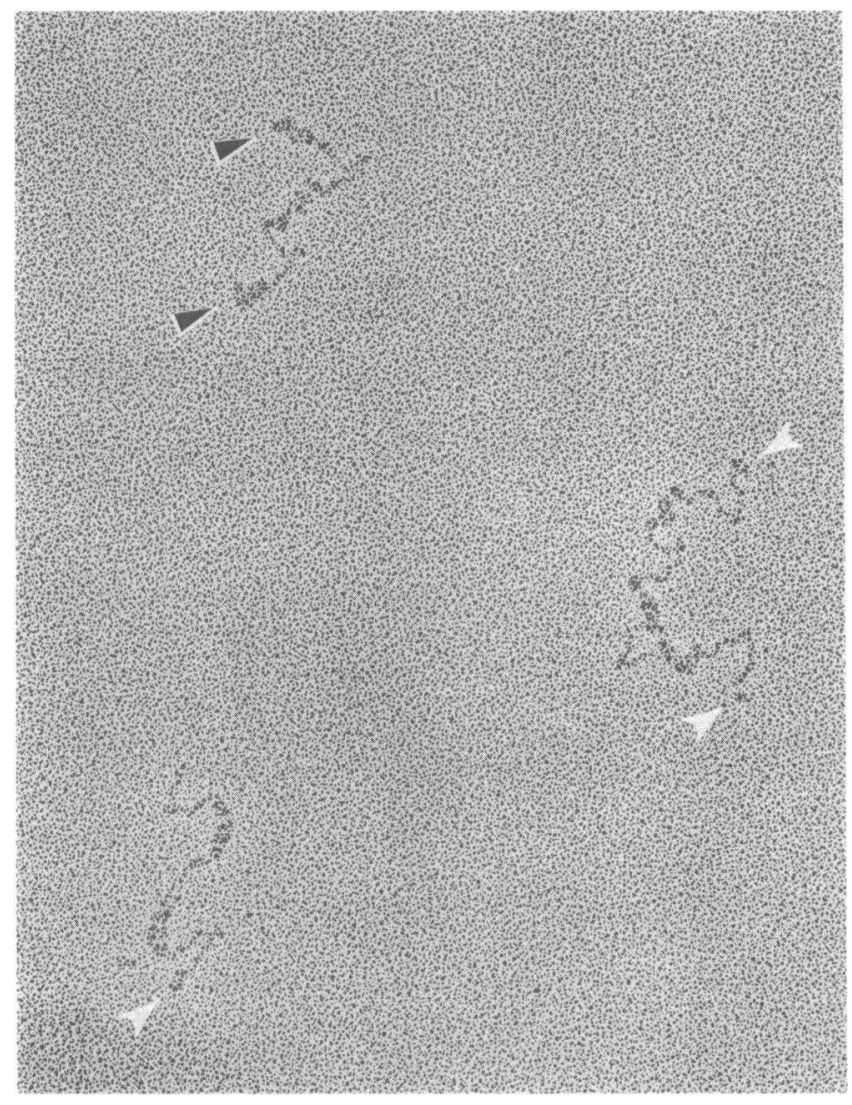

Figure 9. Electron micrograph $(\times 142,000)$ of rotary shadowed $\mathrm{vWf}$ molecules displaying different end morphologies. This sample was from a $1 \%$ agarose column fraction and was not treated with plasmin. Black arrows show globular end domains that are relatively large and white arrows indicate those that are relatively small (compare with normal protomer end in Fig. $7 \mathrm{D}$ ).
$125 \mathrm{~nm}$ diameters, respectively. The $\mathrm{vWf}$ molecules are flexible coiled strands, so their overall size or extent is probably larger than indicated by this calibration (31). A more definitive measure of the size in solution is the radius of gyration. A recent light scattering study has determined a weight-averaged radius of gyration of $90 \mathrm{~nm}$ (32). This would correspond to a sphere of 230 $\mathrm{nm}$ in diameter if the protein is distributed uniformly or randomly. These hydrodynamic and scattering data are therefore completely consistent with the compact forms we observe in the electron microscope specimens.

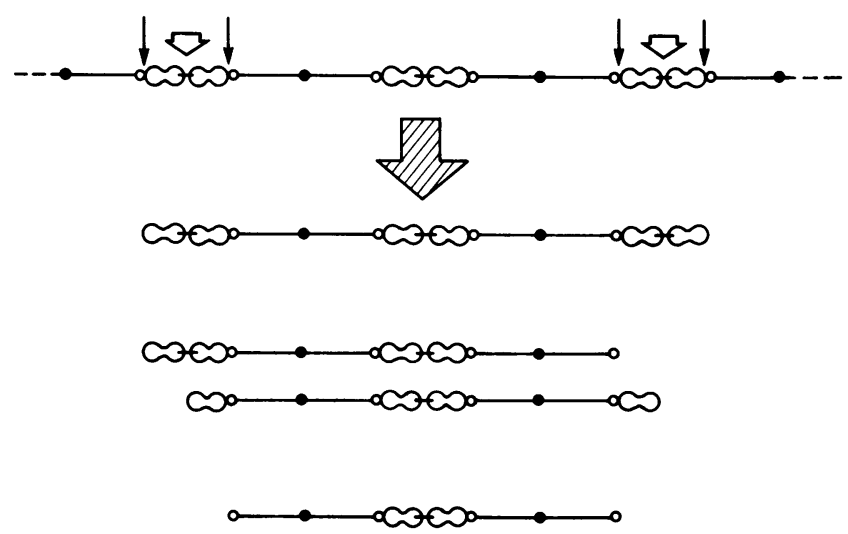

Figure 10. Model for fragmentation of vWf multimer that accounts for the observed variant ends and triplet banding pattern. Thin black arrows show proposed fragmentation sites. Intersubunit disulfide bonds within protomers are depicted by $(\bullet)$ and between protomers are depicted by short horizontal lines connecting globular ends. Short broad arrows mark protomer polymerization sites. Possible products of fragmentation with variant ends or products of normal polymerization (those with single large globular ends) are shown below large striped arrow for the case of a "two-mer." 


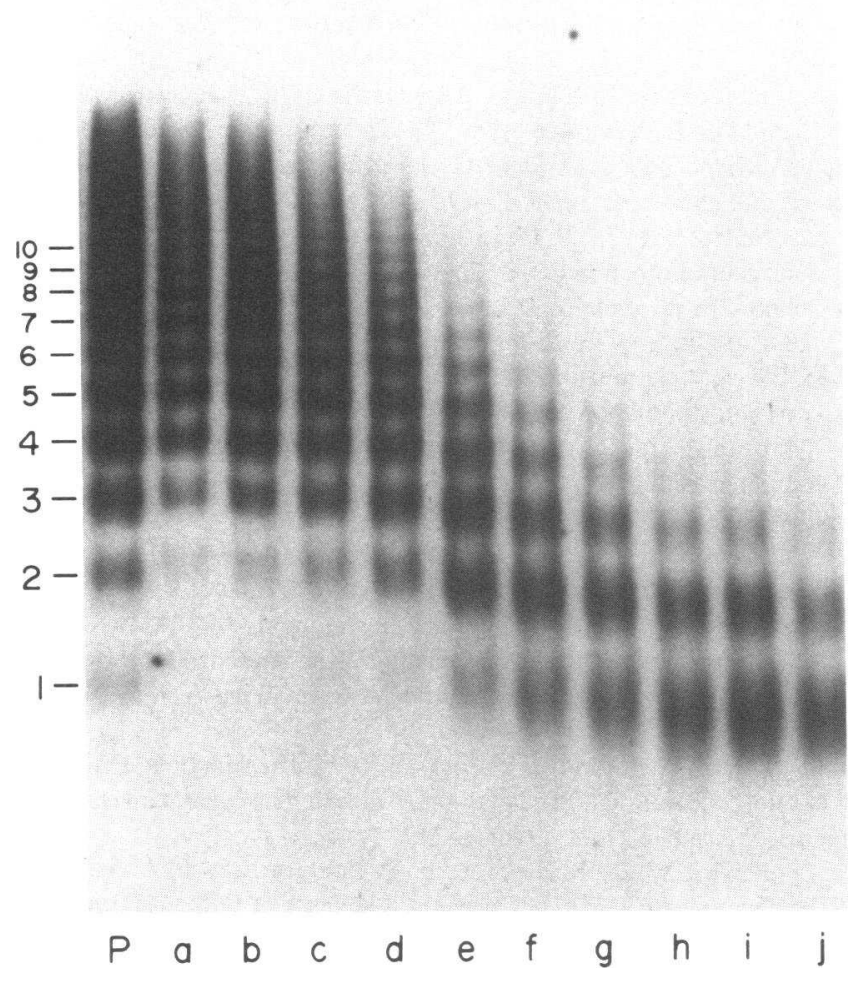

Figure 11. Plasmin digestion of vWf from fresh plasma. Radioimmunoblot of SDS- $0.8 \%$ agarose gel of: $P$, whole plasma $(0.5 \mu 1) ; a$, vWf purified as in Fig. 1 but from plasma containing 5 mM EDTA in addition to citrate, aprotinin, and DFP. Same vWf preparation as in $a$ was digested with plasmin as in Methods for $3 \mathrm{~min}(b), 10 \mathrm{~min}(c), 20 \mathrm{~min}$ $(d), 1 \mathrm{~h}(e), 2 \mathrm{~h}(f), 3 \mathrm{~h}(\mathrm{~g}), 4 \mathrm{~h}(\mathrm{~h}), 5 \mathrm{~h}(i)$, and $7.5 \mathrm{~h}(\mathrm{j})$. Several multimers are identified on the left side by the number of protomers they contain. Arrow at top indicates origin of gel lanes.

The smaller vWf multimers in the conventional preparation appeared by electron microscopy to be less tangled or coiled and were therefore more suitable for substructural analysis. To obtain a more homogeneous sample of small multimers we therefore refractionated vWf purified from FVIII/vWf concentrates (Fig. 4) and obtained subpopulations with decreasing mean weights (Fig. 5) that correlated with a decrease in multimer lengths as seen by electron microscopy (Fig. 6).

The substructure of vWf is seen most clearly in Fig. 7, which contains a gallery of the best images and a model that summarizes our knowledge about the protomer itself. Our model for the overall structure of the protomer is reminiscent of the trinodular fibrinogen molecule $(27,33)$; however, the molecular dimensions of the vWf protomer are quite different. Although it is more than twice as long as the fibrinogen molecule, its central nodule is $25-35 \%$ smaller in volume than fibrinogen Fragment $E$ and corresponds to $\sim 35,000 \mathrm{~mol}$ wt (assuming a partial specific volume of $0.72 \mathrm{ml} / \mathrm{g}$ ). The filamentous domains extending out from this twofold axis of symmetry are each $\sim 34 \mathrm{~nm}$ long, have a diameter of $\sim 2 \mathrm{~nm}$, and are similar to fibronectin in appearance as previously noted (21), but measurably smaller in width. Using the mass per unit length of fibronectin (34-36) permits a maximum estimate of $106,000 \mathrm{~mol} \mathrm{wt}$ for each of these rod domains. Each globular end of the vWf protomer is roughly half the size of the globular interprotomeric region described in Results, namely about 1.75 times that of fibrinogen Fragment $D$, or $\sim 150,000 \mathrm{~mol} \mathrm{wt}$ assuming the same specific volume $(0.72 \mathrm{ml} /$ g). These calculations give an estimate of $550,000 \mathrm{~mol} \mathrm{wt}$ for the total protomer molecular weight. Based on the above considerations and the fact that the vWf protomer is morphologically compatible with a dimer, it appears that the protomer consists of two $240,000-\mathrm{mol}$ wt subunits; i.e., the characteristic subunit of fully reduced $\mathrm{vWf}$. Although a dimeric repeating unit was proposed by Perret et al. (14), other models with four to eight subunits per $v W f$ protomer have also been suggested (2). Recent work of Wagner and Marder (37) has identified a dimer of the 240,000-mol wt subunit as being an intermediate in endothelial cell assembly of multimeric vWf. Interestingly, Titani et al. (17) have recently reported that Staphylococcus aureus V8 protease cleaves native $\mathrm{vWf}$ into amino- and carboxyl-terminal fragments with reduced molecular weights of 150,000 and $100,000 \mathrm{~mol}$ $w t$, respectively. Electron microscopy of these fragments shows that they correspond to the globular and rod domains, respectively, of our proposed subunit structure (38). Based on all of the above observations, polymerization of the $\mathrm{vWf}$ protomers occurs at the large globular domains in an end-to-end fashion. Since the protomer is structurally nonpolar (i.e., the two subunits face away from their point of contact at the center of the protomer), the vWf polymer is also nonpolar.

We made several attempts to isolate a homogeneous population of single vWf protomers. Disulfide bond reduction of $\mathrm{vWf}$ resulted in the complete loss of the filamentous structure of $\mathrm{vWf}$ and in the formation of large, uninterpretable aggregates in electron microscope preparations (21). Fractionation of vWf multimers on sizing columns gave subpopulations containing only two or three multimer sizes, but individual protomers were only a small percentage of the total vWf present in any of these fractions (Fig. 5). However, we did obtain additional support for our identification of the protomeric structure by finding that, as predicted by other gel electrophoretic studies (23), plasmin digestion of fractionated multimers yielded molecules with the characteristic appearance of $\mathrm{vWf}$ protomers (Fig. $8 \mathrm{~B}$ ). Although plasmin degradation of native $\mathrm{vWf}$ multimers does not generate the triplet banding pattern (Fig. 11), some other proteinase may be responsible. Some have suggested that calcium-dependent protease from platelets gives rise to lower molecular weight $\mathrm{vWf}$ multimers (39). However, in preliminary studies (data not shown), incubation of $\mathrm{vWf}$ with lysed platelets in the presence or absence of EDTA did not generate the triplet band pattern as judged by radioimmunoblots. We present two new lines of data that suggest a model (Fig. 10) for the origin of the triplet bands and size heterogeneity: (a) different preparations of $\mathrm{vWf}$ show no morphological difference in internal substructure, despite major variations in size and in the extent of their triplet band pattern on radioimmunoblots, and $(b)$ morphological differences are consistently observed at the globular end domains of these vWf multimers.

In conclusion, our results on the substructure of $v W f$ suggest that $\mathrm{vWf}$ multimers are formed by the linear polymerization of dimeric protomers in an end-to-end fashion. Since linear polymerization alone cannot explain the variant end morphologies that we observed, these are probably produced by fragmentation of large multimers at points within protomers. An appealing hypothesis is that circulating multimers may be derived proteolytically from a larger precursor by the action of a proteinase, which may be either plasma-derived or integral to platelets and/ or endothelial cells. 


\section{Acknowledgments}

This work was supported in part by National Heart, Lung, and Blood Institute grants HL-15615 and HL-23454.

\section{References}

1. Hoyer, L. W. 1981. The factor VIII complex: structure and function. Blood. 58:1-13.

2. Zimmerman, T. S., Z. M. Ruggeri, and C. A. Fulcher. 1983. Factor VIII/von Willebrand factor. In Progress in Hematology, Vol. XIII. E. B. Brown, editor. Grune \& Stratton, Inc., New York. 279-309.

3. McKee, P. A. 1981. Observations on structure-function relationships of human antihemophilic/von Willebrand factor protein. Ann. NY. Acad. Sci. 370:210-226.

4. Hoyer, L. W., R. de los Santos, and J. R. Hoyer. 1973. Antihemophilic factor antigen. Localization in endothelial cells by immunofluorescent microscopy. J. Clin. Invest. 52:2737-2744.

5. Jaffe, E. A., L. W. Hoyer, and R. L. Nachman. 1973. Synthesis of antihemophilic factor antigen by cultured human endothelial cells. $J$. Clin. Invest. 52:2757-2764.

6. Zimmerman, T. S., J. Roberts, and T. S. Edgington. 1975. FactorVIII-related antigen: multiple molecular forms in human plasma. Proc. Natl. Acad. Sci. USA. 72:5121-5125.

7. Gralnick, H. R., B. S. Coller, and Y. Sultan. 1975. Studies of the human factor VIII/von Willebrand factor protein. J. Clin. Invest. 56: 814-827.

8. Counts, R. B., S. L. Paskell, and S. K. Elgee. 1978. Disulfide bonds and the quaternary structure of factor VIII/von Willebrand factor. $J$. Clin. Invest. 62:702-709.

9. Hoyer, L. W., and J. R. Shainoff. 1980. Factor-VIII-related protein circulates in normal human plasma as high molecular weight multimers. Blood. 55:1056-1059.

10. Ruggeri, Z. M., and T. S. Zimmerman. 1980. Variant von Willebrand disease. Characterization of two subtypes by analysis of multimeric composition of factor VIII/von Willebrand factor in plasma and platelets. J. Clin. Invest. 65:1318-1325.

11. Meyer, D., B. Obert, G. Pietu, J. M. Lavergne, and T. S. Zimmerman. 1980. Multimeric structure of factor VIII/von Willebrand factor in von Willebrand's disease. J. Lab. Clin. Med. 95:590-602.

12. Martin, S. E., V. J. Marder, C. W. Francis, and G. H. Barlow. 1981. Structural studies on the functional heterogeneity of von Willebrand protein polymers. Blood. 57:313-323.

13. Ruggeri, Z. M., and T. S. Zimmerman. 1981. The complex multimeric composition of factor VIII/von Willebrand factor. Blood. 57: 1140-1143.

14. Perret, B. A., M. Furlan, and E. A. Beck. 1979. Studies in factor VIII-related protein. II. Estimation of molecular size differences between factor VIII oligomers. Biochim. Biophys. Acta. 578:164-174.

15. Shapiro, G. A., J. C. Andersen, S. V. Pizzo, and P. A. McKee. 1973. The subunit structure of normal and hemophilic factor VIII. $J$. Clin. Invest. 52:2198-2210.

16. Legaz, M. E., G. Schmer, R. B. Counts, and E. W. Davie. 1973. Isolation and characterization of human factor VIII (antihemophilic factor). J. Biol. Chem. 248:3946-3955.

17. Titani, K., L. H. Ericsson, S. Kumar, H. Dorsam, M. W. Chopek, and K. Fujikawa. 1984. Chemical characterization of human von Willebrand factor. Circulation. 70:837a. (Abstr.)

18. Mannucci, P. M., R. Lombardi, F. I. Pareti, S. Solinas, M. G. Mazzucconi, and G. Mariani. 1983. A variant of von Willebrand's disease characterized by recessive inheritance and missing triplet structure of von Willebrand factor multimers. Blood. 62:1000-1005.

19. Zimmerman, T. S., and Z. M. Ruggeri. 1982. von Willebrand's disease. In Progress in Hemostasis and Thrombosis, Vol. 6. T. H. Spaet, editor. Grune \& Stratton, Inc., New York. 203-236.

20. Zimmerman, T. S., J. A. Dent, A. B. Federici, Z. M. Ruggeri,
I. M. Nilsson, L. Holmberg, C. A. Abilgaard, and L. H. Nannini. 1984. High resolution $\mathrm{NaDodSO}_{4}$-agarose electrophoresis identifies new molecular abnormalities in von Willebrand disease. Clin. Res. 32:II-501a. (Abstr.)

21. Ohmori, K., L. J. Fretto, R. L. Harrison, M. E. P. Switzer, H. P. Erickson, and P. A. McKee. 1982. The electron microscopy of human factor VIII/von Willebrand factor glycoprotein: effect of reducing reagents on structure and function. J. Cell Biol. 95:632-640.

22. Sodetz, J. M., S. V. Pizzo, and P. A. McKee. 1977. Relationship of sialic acid to function and in vivo survival of human factor VIII/von Willebrand factor protein. J. Biol. Chem. 252:5538-5546.

23. Hamilton, K. K., L. J. Fretto, D. S. Grierson, and P. A. McKee. 1985. Effects of plasmin on von Willebrand factor multimers. Degradation in vitro and stimulation of release in vivo. J. Clin. Invest. 76:261-270.

24. Lucas, M. A., L. J. Fretto, and P. A. McKee. 1983. The binding of human plasminogen to fibrin and fibrinogen. J. Biol. Chem. 258: 4249-4256.

25. Laemmli, U. K., and M. Favre. 1973. Maturation of the head of bacteriophage T4. I. DNA packaging events. J. Mol. Biol. 80:575-599.

26. Merril, C. R., D. Goldman, S. A. Sedman, and M. H. Ebert. 1981. Ultrasensitive stain for proteins in polyacrylamide gels shows regional variation in cerebrospinal fluid proteins. Science (Wash. DC). 211:1437-1438.

27. Fowler, W. E., and H. P. Erickson. 1979. The trinodular structure of fibrinogen: confirmation by both shadowing and negative stain electron microscopy. J. Mol. Biol. 134:241-249.

28. Fowler, W. E., L. J. Fretto, H. P. Erickson, and P. A. McKee. 1980. Electron microscopy of plasmic fragments of human fibrinogen as related to trinodular structure of the intact molecule. J. Clin. Invest. 66:50-56.

29. Fowler, W. E., and U. Aebi. 1983. Preparation of single molecules and supramolecular complexes for high-resolution metal shadowing. $J$. Ultrastruct. Res. 83:319-334.

30. Reynolds, J. A., Y. Nozaki, and C. Tanford. 1983. Gel-exclusion chromatography on S1000 Sephacryl: application to phospholipid vesicles. Anal. Biochem. 130:471-474.

31. Nozaki, Y., N. M. Schechter, J. A. Reynolds, and Tanford. 1976. Use of gel chromatography for the determination of the Stokes radii of proteins in the presence and absence of detergents: a reexamination. Biochemistry. 15:3884-3890.

32. Binnie, C. G., R. H. Wagner, H. Cooper, and J. Hermans. 1985. Conformation of bovine von Willebrand factor in solution as studied by light scattering. Thromb. Haemostasis. 54:75. (Abstr.)

33. Hall, C., and H. Slayter. 1959. The fibrinogen molecule: its size, shape, and mode of polymerization. J. Biophys. Biochem. Cytol. 5:1115.

34. Erickson, H. P., N. Carrell, and J. McDonagh. 1981. Fibronectin molecule visualized in electron microscopy: a long, thin, flexible strand. J. Cell Biol. 91:673-678.

35. Engel, J., E. Odermatt, A. Engel, J. A. Madri, H. Furthmayr, H. Rohde, and R. Timpl. 1981. Shapes, domain organizations and flexibility of laminin and fibronectin, two multifunctional proteins of the extracellular matrix. J. Mol. Biol. 150:97-120.

36. Odermatt, E., J. Engel, H. Richter, and H. Hormann. 1982. Shape, conformation and stability of fibronectin fragments determined by electron microscopy, circular dichroism and ultracentrifugation. J. Mol. Biol. 159:109-123.

37. Wagner, D., and V. Marder. 1984. Biosynthesis of von Willebrand protein by human endothelial cells: processing steps and their intracellular localization. J. Cell. Biol. 99:2123-2130.

38. Fretto, L. J., W. E. Fowler, K. K. Hamilton, and P. A. McKee. 1985. Substructure of human von Willebrand factor protomer: fragmentation by plasmin and S. Aureus V8 protease. Thromb. Haemostasis. 54:59. (Abstr.).

39. Kunicki, T. J., R. R. Montgomery, and J. R. Schullek. 1984. Cleavage on human von Willebrand factor by platelet calcium-activated protease. Blood. 65:352-356. 